\title{
Determinación estructural de la periodización táctica en el baloncesto femenino de la Espoch sede Morona Santiago Ecuador.
}

Structural determination of tactical periodization in women's basketball at the Espoch headquarters Morona Santiago Ecuador.

Alberto Alzola Tamayo. ${ }^{1}$, Danilo Ortiz Fernández. ${ }^{2}$, Mario Enrrique Vaca. ${ }^{3} \&$ Santa Alzola Tamayo. ${ }^{4}$

Recibido: 27-04-2019 / Revisado: 26-05-2019 /Aceptado: 28-06-2019/ Publicado: 15-07-2019

\section{Resumen.}

DOI: https://doi.org/10.33262/cienciadigital.v3i3.1.710

Para dar solución a las insuficiencias en el proceso de aprendizaje de la táctica de juego que se manifiesta en los estudiantes deportistas del equipo de baloncesto femenino de la Escuela Superior Politécnica de Chimborazo Extensión morona Santiago. El universo de trabajo estuvo constituido por 15 deportistas. Esta investigación se realizó con el objetivo de evaluar el efecto de la aplicación de la metodología periodización táctica en el entrenamiento, cuya preocupación máxima es el jugo que un equipo pretende producir en la competición. Partiendo del modelo de juego, modelando a través de los cuatros momentos que engloban el juego (organización ofensiva, organización defensiva, transición de ataque a defensa y de defensa a ataque), consiguiendo alcanzar una adaptación específica y de calidad. Lo aprendido por este camino (periodización táctica) propicio un aumento en el accionar del juego global, así como una consolidación y perfeccionamiento del proceso de aprendizaje de la táctica, garantizando que aflore la creatividad en los deportistas.

Palabras claves: Periodización táctica, Proceso de aprendizaje del baloncesto

\begin{abstract}
To solve the shortcomings in the learning process of the game tactics that is manifested in student athletes of the women's basketball team of the Higher Polytechnic School of Chimborazo Morona Santiago extension. The universe of work consisted of 15 athletes. This research was carried out with the objective of evaluating the effect of the application of tactical periodization methodology in

\footnotetext{
${ }^{1}$ Escuela Superior Politécnica de Chimborazo Sede Morona Santiago. Ecuador. alberto.alzola@espoch.edu.ec

${ }^{2}$ Escuela Superior Politécnica de Chimborazo Sede Morona Santiago. Ecuador. danilo.ortiz@espoch.edu.ec

${ }^{3}$ Escuela Superior Politécnica de Chimborazo Sede Morona Santiago. Ecuador. maria.vaca@espoch.edu.ec

${ }^{4}$ Escuela Superior Politécnica de Chimborazo Sede Morona Santiago. Ecuador. Santa. tamayo@espoch.edu.ec
} 


\section{C) Ciencia

training, whose maximum concern is the juice that a team intends to produce in the competition. Starting from the game model, modeling through principles, subprinciples, subsubprinciples of the game that form it, achieving a specific adaptation and quality. What has been learned in this way (tactical periodization) favored an increase in the action of the global game, as well as a consolidation and improvement of the learning process of the tactics, guaranteeing that creativity arises in the athletes.

Keywords: Tactical periodization, Basketball learning process

\section{introducción:}

El entrenamiento deportivo ha estado influenciado por la tendencia mecanicista del ser humano. A pesar que siempre se aluda necesidad de integrar todos los aspectos del entrenamiento y se propongan concepciones holísticas (integradas), la estructura sigue siendo visión cartesiana que concibe a los organismos prácticamente como maquinas construidas por diferentes partes.(Torrents,2005).

\section{Marco Teórico}

La teoría de los sistemas dinámicos ha evidenciado que los organismos vivos funcionan como un todo y que interactúan con el medio.

Carlota Torrents, estudio la teoría de los sistemas dinámicos, no cuestiono la efectividad de los métodos tradicionales de entrenamiento basados en repeticiones de ejercicios y cargas crecientes de trabajo, sino que platea que se puede conseguir los mismos resultados de forma más breve, menos agresiva y más enriquecedora para el deportista. No obstante recuerda que hay aspectos del entrenamiento tradicional que se consideran criticables y perjudiciales para el deportista y que son mejorables si consideramos al individuo como un ser global que interactúa consigo mismo y con su entorno.

Esta propuesta puede aplicarse a todo tipo de deporte. En el caso del baloncesto la variación constante de las condiciones del juego obliga a aprender a adaptarse a esta variabilidad la cual será de gran utilidad.

Mediante esta forma de entrenar siguiendo la teoría de los sistemas dinámicos el deportista no tendrá una idea preconcebida del movimiento, lo que facilitara que este más atento a las sensaciones y fuerzas reactivas que experimenta en su interior, por lo tanto más abierto a entender cómo se organiza su sistema. El profesor podrá aprender también del deportista y de los movimientos que este descubre para dar solución a la tarea.

Seirul - lo afirma en el siglo xx el entrenamiento ha estado dominado por prácticas cuantitativas de ejercicios analíticos en secuencias lineales progresivas cuyo objetivo era construir jugadores para satisfacer las exigencias del modelo conductista (mente) mecanicista (cuerpo) que se reproducía en una serie de factores ajenos al deportista 
(reglamento, exigencias de la competición, sociedad) y trabajando por separado la mente y el cuerpo.

De manera que pronostica que el siglo XXl el deporte se desarrollara de manera integral donde la enseñanza- entrenamiento del cuerpo y la mente será un proceso único de optimización del deportista. El nuevo paradigma busca la (auto-modelación) del deportista, la cual se logra por una serie de propuestas que parten del deportista (instauración de habilidades en competencia, formación propia de imágenes y logro de conocimiento mediante la práctica.

Esta nueva concepción sistémica del jugador como un 'todo" nos dará indicios sobre las condiciones en el deportista deberá desarrollar su entrenamiento. Este nuevo paradigma es ideal para deportes de equipos como el baloncesto donde la interacción es continua entre compañeros, rivales, objeto y se necesita altos niveles de auto estructuración. Siendo la enseñanza entrenamiento un hecho único. Seiru-lo afirma que se deben construir ejercicios que proporcionen alta variabilidad y globalidad lejos de aquellas obsoletas tareas analíticas, cerradas y repetitivas. También manifiesta que el rendimiento no podrá ser evaluado desde criterios cuantitativos ajenos al sujeto, sino a través de propuestas que parten del deportista.

Paralelamente a esto Cano plantea que las cualidades y capacidades físicas son indivisibles, han de estar unidas en el entrenamiento. Consideramos que el nexo de unión de todos estos elementos inseparables, es el compromiso para defender, compromiso para contraatacar, compromiso para ser solidarios en los esfuerzos (ayudar y recobrar) en conclusión compromiso con el pensamiento colectivo.

Según Raspaport (1980) define comunidad como un grupo de personas que viven en un área geográfica determinada y cuyos miembros comparten actividades e intereses comunes donde pueden o no cooperar para la solución de problemas. Esta definición vincula elementos funcionales y estructurales, como elemento estructural se define un grupo que vive en determinada área geográfica. Los elementos funcionales se refieren a aquellos que aglutinan a sus integrantes y sirven de base a su movilización en torno a tareas comunes en nuestro caso el baloncesto.

Sobre la base de lo expresado anteriormente es preciso definir los problemas actuales que presenta el proceso de enseñanza-entrenamiento del baloncesto en la comunidad del barrio norte perteneciente al cantón Morona con una población de 18984 habitantes. Sus límites son al norte con la calle Don Bosco al sur con la calle José Pintado detrás del estadio Tito Navarrete Álava Provincia Morona Santiago. Teniendo en cuenta las características de esta comunidad para la selección de la muestra la técnica de muestreo aleatoria fue escogida debido a la necesidad de extraer grupos etarios que la componen.

\section{Un acercamiento a la periodización táctica}

La periodización táctica surge hace más de 30 años de la cabeza de Víctor Frade, cuando a través de experiencias que le iban ocurriendo comienza a cuestionarse las metodologías de 
entrenamiento existentes hasta el momento. Frade interiorizo el juego que producen los deportes de equipo dentro de ellos el futbol y el baloncesto no puede entenderse desde el pensamiento científico clásico (analítico y desconceptualizado) dado su globalidad, imprevisibilidad y estado no lineal, decide buscar en teorías más adecuadas a los tipos de problemas que estos plantean llegando a teorías sistémicas que junto a ciertos principios metodológicos configuro la periodización táctica.

El termino periodización táctica es una metodología que define el jugar de un equipo y crea una identidad que dirige y orienta a todos a los que a ella pertenecen haciendo aflorar una especificidad propia.

\section{La necesidad de una metodología específica para el entrenamiento del baloncesto.}

Metodología de la enseñanza es el conjunto de acciones que configuran la conducta del entrenador en sus cometidos de enseñanza con el fin de lograr de forma más eficiente los objetivos del entrenamiento previstos (Sánchez Buñuelos)

La necesidad de buscar una metodología que fuera netamente para el baloncesto y que tomara distancia de los ejercicios analíticos y desconceptualizados de la realidad de juego de baloncesto.

En la periodización táctica es fundamental en el proceso el modelo de juego que permite la creatividad de los jugadores pues como refiere Fleitas,S.(2004), el modelo de juego será más rico cuando más posibilite a los jugadores ampliar su propia creatividad y talento en el juego.

De manera que el modelo de juego es un conjunto de comportamientos que el entrenador pretende que su equipo manifieste de forma regular y sistemática en los cuatro momentos de juego (organización ofensiva, organización defensiva y las dos fases transitorias de una organización hacia otra. Estos comportamientos son reproducidos como principios, subprincipios y subsubprincipos. Cuando logramos que estos se articulen entre sí, proporcionan una forma de jugar lo que vendría a ser el estilo del equipo el cual deberá ser mejorado día a día sin perder la identidad y permitiendo la creatividad de los jugadores.

La periodización táctica respeta el principio de "entereza inquebrantable" del juego, globalizando en cada ejercicio las cuatro dimensiones que forman al baloncesto.

La supradimensión táctica es por tanto, quien regula todo el proceso. Lo táctico no es ni físico, ni técnico, ni psicológico, arrastra a estas dimensiones se carga solo, llega convoyado. Esta supradimensión es la que permitirá la interacción entre todas las dinámicas (Delgado Méndez, 2012).

\section{Morfociclo Patrón.}

La periodización táctica defiende la utilización de un morfociclo patrón a lo larga de todo el ciclo competitivo. Es la forma en que se organizan los entrenamientos semanalmente. Para ello se apoya en el encuentro anterior y el encuentro posterior. La diferencia entre el 
microciclo y el morfociclo es que el primero solo son sesiones de determinado número que se programan y asumen diferentes formas y diferentes objetivos. Sin embargo el morfociclo patrón busca dar forma al modelo de juego cocinando y modelando algunos procesos.

Pese a que los morfociclos a lo largo de una temporada son idénticos, no quiere decir que sus contenidos también lo sean.

\section{Comportamiento de la concentración en los entrenamientos.}

En el baloncesto moderno es necesaria la concentración durante los 40 minutos de juego, antes, durante y después del mismo en los entrenamientos, en las charlas técnicas, para ello debe entrenarse de forma específica para el modelo de juego y preparar al jugador para el partido operacionalizando el juego de forma previa al partido y post partido. La concentración de ejercicios donde los jugadores tienen que pensar mucho, ejercicios de complejidad reciente que le obligue a una concentración permanente. Para entrenar la concentración táctica es necesario hacerla con ejercicios que simulen nuestro modelo de juego.

\section{Análisis de los resultados}

En los análisis de los datos aportados por las observaciones del instrumento para medir los resultados se observa una tendencia a la categoría siempre y casi siempre lo que indica que en los estudiantes influyo positivamente los ejercicios.

Tabla No1 Resultados
S: SIEMPRE
CS: CASI SIEMPRE
PV:POCAS VESES
$\mathrm{N}$ : NUNCA

\begin{tabular}{|l|l|l|l|l|l|l|l|l|}
\hline & $\mathrm{S}$ & $\%$ & $\mathrm{CS}$ & $\%$ & $\mathrm{PV}$ & $\%$ & $\mathrm{~N}$ & $\%$ \\
\hline $\begin{array}{l}\text { ORGANIZACIÓN } \\
\text { OFENSIVA }\end{array}$ & 11 & 73,3 & 3 & 20 & 1 & 6.6 & - & - \\
\hline $\begin{array}{l}\text { ORGANIZACIÓN } \\
\text { DEFENSIVA }\end{array}$ & 4 & 26,6 & 3 & 20 & 4 & 26,6 & 4 & 26,6 \\
\hline $\begin{array}{l}\text { TRANSICIÓN } \\
\text { ATAQUE / DEFENSA }\end{array}$ & 7 & 46,6 & 6 & 40 & 2 & 13,3 & -- & - \\
\hline $\begin{array}{l}\text { TRANSICIÓN } \\
\text { DEFENSA / ATAQUE }\end{array}$ & 12 & 80 & 2 & 13,33 & 1 & 6,6 & - & - \\
\hline
\end{tabular}


Después de aplicados los ejercicios se pudo demostrar que todos los indicadores se movieron ascendentemente, a partir de transición defensa - ataque, organización ofensiva las cuales fueron de interés paras los deportistas, la mayor dificultad radicó en la organización defensiva. A continuación se exponen las particularidades de este proceso de implementación y sus efectos. Los ejercicios que incentivaron la periodización táctica para favorecer la el proceso de consolidación y perfeccionamiento se fundamentan en el juego global en baloncesto se sustenta la necesidad de un diagnóstico integral y permanente de la necesidad de una mayor compromiso en la defensa, haciendo énfasis en los entrenamientos de esta fase de juego para lograr la transformación integral de los deportistas y su papel activo y protagónico en el proceso de enseñanza- aprendizaje del baloncesto.

Muchas fueron las experiencias y logros obtenidos cuando se comenzaron a aplicar los ejercicios elaborados en función de hacer brotar en los deportistas la creatividad que estén aptos para producir el juego.

El objetivo de incorporar ejercicios en situaciones de juego no fue por el mero hecho de entretenerlos, exclusivamente, sino para lograr la concientización de la necesidad que tienen de observar los estímulos del entorno y la lucha de contrarios, algo fundamental en el desarrollo del pensamiento técnico - táctico dentro del juego.

En los ejercicios pertenecientes a la dimensión ataque - defensa, se pudo comprobar que no pasaron inadvertidos, se le añadieron ingredientes interesantes que contribuyeron a la motivación y todos mejoraron sus niveles en cuanto a la anticipación en la defensa y el salto y cambio.

. En correspondencia con la dimensión organización ofensiva el desenvolvimiento fue superior a la medición inicial pero la tendencia se movió a la categoría de siempre con 73,3 \% que representa 11 deportistas y casi siempre con 20.0 que representa 3 deportistas esto quiere decir que no existe un total conocimiento del sistema ofensivo específicamente en la conexión de juego continuar jugando después de la transición ya que los estudiantes 5 y 6 no mejoraron sus resultados en el test final.

Se presentaron varias dificultades para el desarrollo y logro de los ejercicios pertenecientes a la dimensión transición ataque - defensa porque hubo que explicar en qué consistía este ejercicio y esto rebasó el tiempo disponible para la parte teórica y la disposición de los estudiantes al no 


\section{- Cíncia Digital}

estar acostumbrados a comprometerse con la mayor anticipación al regreso al balance defensivo la mayor cantidad de jugadores después de perder un balón o fallar un tiro. Fue fácil sistematizar el dominio de la transición defensa - ataque basado en cuidar detalles como el corrido por los carriles y mantención de espacios entre jugadores donde 12 jugadoras que representa el $80 \%$ se movió a la categoría de siempre.

A continuación se hará referencia a los resultados comparativos obtenidos antes y después de aplicados los ejercicios conjuntamente con las dimensiones propuestas.

Tabla N2: Desarrollo y logro de los ejercicios

\section{S:SIEMPRE CS: CASI SIEMPRE PV:POCAS VESES N: NUNCA}

\begin{tabular}{|c|c|c|c|c|c|c|c|c|c|c|c|c|c|c|c|c|}
\hline \multirow[t]{2}{*}{ Dimensiones } & \multicolumn{8}{|c|}{ Primera medición } & \multicolumn{7}{|c|}{ Segunda medición } & \\
\hline & $\mathrm{S}$ & $\%$ & C.S & $\%$ & P.V & $\%$ & $\mathrm{~N}$ & $\%$ & $\mathrm{~S}$ & $\%$ & C.S & $\%$ & P.V & $\%$ & $\mathrm{~N}$ & $\%$ \\
\hline $\begin{array}{l}\text { ORGANIZACIÓN } \\
\text { OFENSIVA }\end{array}$ & 1 & 9.0 & 1 & 9.0 & 7 & 63.6 & 2 & 18.1 & 8 & 72.7 & 2 & 18.1 & 1 & 9.1 & - & - \\
\hline $\begin{array}{l}\text { ORGANIZACIÓN } \\
\text { DEFENSIVA }\end{array}$ & 1 & 9.0 & 1 & 9.0 & 6 & 54.5 & 3 & 27.2 & 10 & 90.9 & 1 & 9.1 & - & - & - & - \\
\hline $\begin{array}{l}\text { TRANSICIÓN } \\
\text { ATAQUE } \\
\text { DEFENSA }\end{array}$ & - & - & 1 & 9.0 & 2 & 18.1 & 8 & 72.7 & 4 & 36.3 & 5 & 45.4 & 1 & 9.1 & 1 & 9.1 \\
\hline $\begin{array}{l}\text { TRANSICIÓN } \\
\text { DEFENSA } \\
\text { ATAQUE }\end{array}$ & - & - & - & - & 2 & 18.1 & 9 & 81.8 & 7 & 63.6 & 1 & 9.1 & 2 & 18.1 & 1 & 9.5 \\
\hline
\end{tabular}

De acuerdo con los antecedentes del problema que afecta en gran medida el proceso de consolidación y perfeccionamiento del juego global en las jóvenes y por tener como única meta la práctica del baloncesto el autor confeccionó un morfociclo patrón teniendo en cuenta que se celebrara un partido semanal.

\section{Tabla N3: Contenidos}

\section{OBJETIVOS/CONTENIDOS PRINCIPALES DE LA SESIÓN DEL LUNES}

Contracciones e interacciones de nuestro jugar

Elevada tensión de contracción

Saltos, choques, arrancadas, lanzamientos, cambios de dirección

Ejercicios con pocos jugadores, poca duración y espacios reducidos

Entrenamiento competitivo con trabajo del metabolismo específico 


\section{$0^{\text {Conatg }}$

\section{CALENTAMIENTO (15 MINUTOS) REPRESENTACIÓN GRÁFICA}

Ejercicio de competición de lanzamientos por parejas. La primera pareja que llegue a 40 puntos (tiros de 3 y de 2 , con un máximo de dos consecutivos en la misma canasta), gana.

Contraataque de 11: ejercicio de 3c2 continuo. Iniciaremos pautas de cómo correr el contraataque y rapidez de las acciones (máximo 8 segundos para atacar y sólo puede tirar quien esté solo). Quien ataca coge el rebote ataca con los dos compañeros en banda. No se realizará excesivas correcciones porque el objetivo principal del ejercicio es alcanzar nivel óptimos físicos y psicológicos para iniciar la parte principal del entrenamiento.

\section{PARTE PRINCIPAL (60 MINUTOS)}

\section{REPRESENTACIÓN GRÁFICA}

Ejercicio de 1c1 por parejas, con tres defensas y tres ataques consecutivos. Una vez atacan y defiendes los dos, hay un cambio de posición dentro de las 4 posiciones exteriores del triple. Se pueden poner limitaciones de bote y puntuamos los puntos anotados de cada uno (competición: 7 minutos)

Ejercicio de 2c2 a partir de situación de bloqueo directo, para trabajar movimiento sin balón y cambios defensivos tras bloqueos (10 minutos)

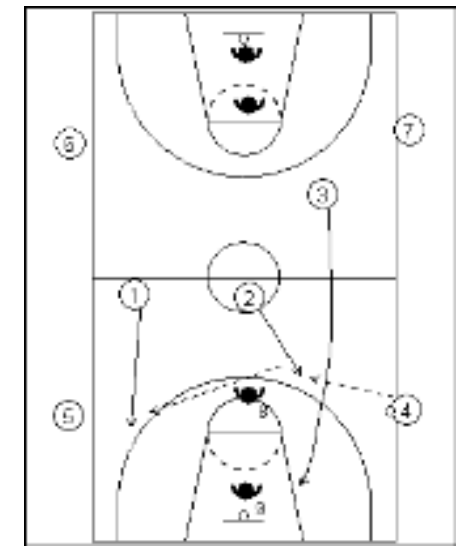

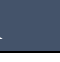


Ejercicio de 2c2 a todo campo para trabajar la defensa del corte y el tráiler central. Premiamos los ataques rápidos (12 minutos)

Ejercicio de $3 \mathrm{c} 3$ en medio a partir de situación de bloqueo directo lateral con el objetivo de trabajar las normas de movimiento sin balón y defensas de ajustes y cambios (15 minutos)

Ejercicio de $3 \mathrm{c} 3$ continuo a todo campo (quien anota sigue atacando), para trabajar los conceptos antes mencionados a nivel ofensivo y defensivos (15 minutos)

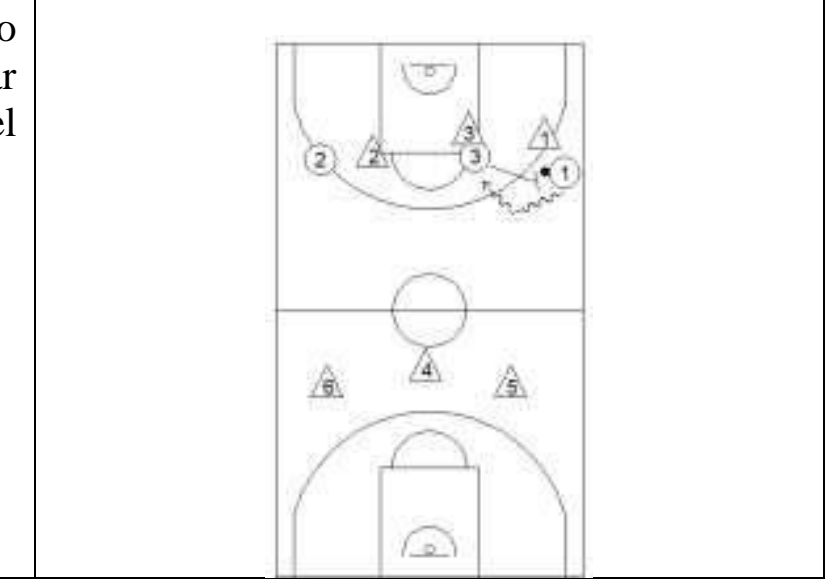

\section{OBJETIVOS/CONTENIDOS PRINCIPALES DE LA SESIÓN DEL MARTES}

Dominancia de las fibras de contracción rápidas

Trabajo de los principios del juego

Entrenamiento con mayor complejidad (mayor interrelación entre compañeros, espacios más amplios, etc.)

Descansos-Intermitencia que permita realizar los ejercicios a máxima intensidad 


\section{Ciencia Digital}

Esta sesión será la más semejante a la propia competición. Como los partidos con mayor trascendencia competitiva se acercan, se realizarán ejercicios en su mayoría de 5 vs 5 con alguna variante, como, por ejemplo, algún equipo con desventaja en el marcador favoreciendo el desgaste emocional.

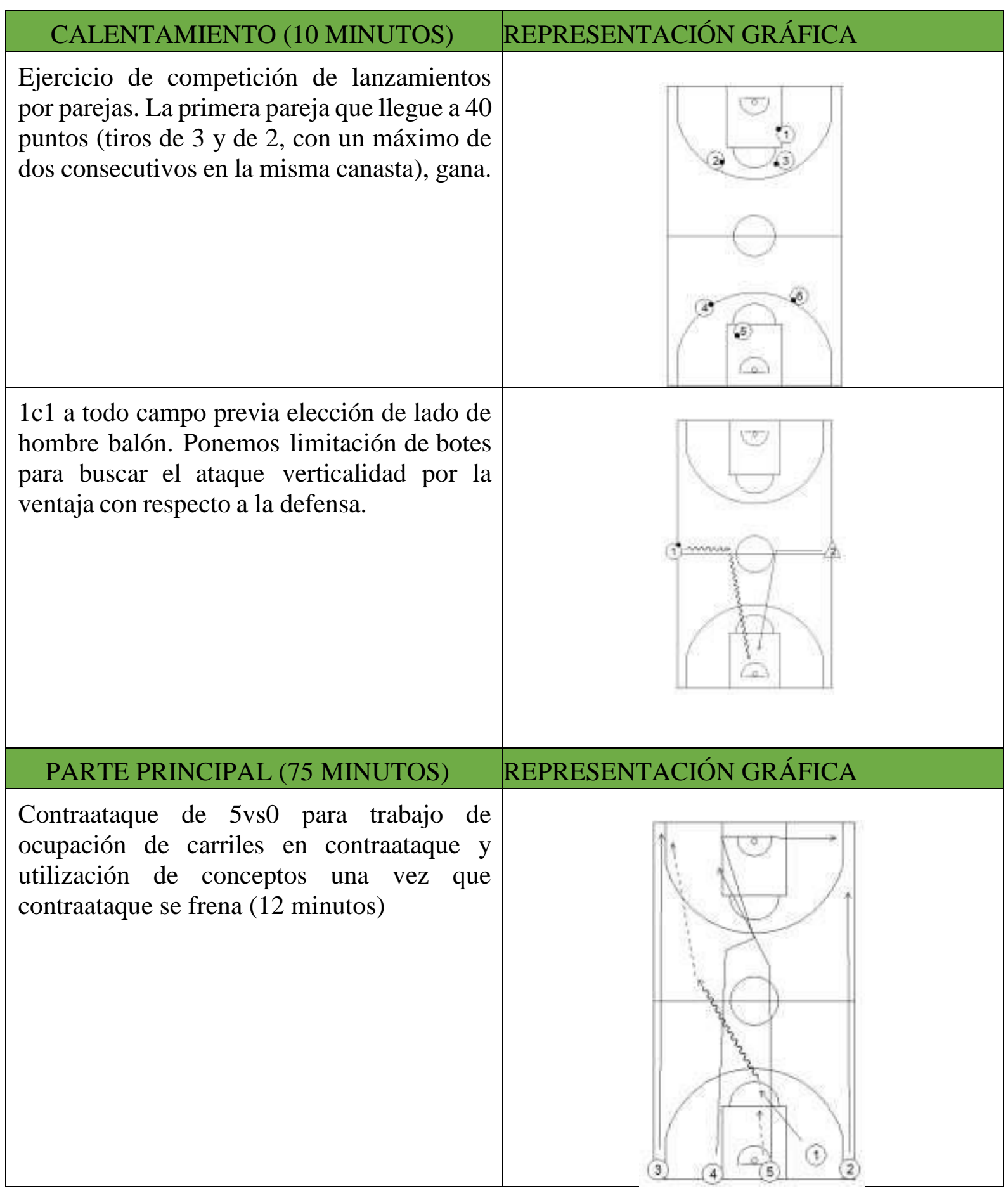




\section{- Ciencia

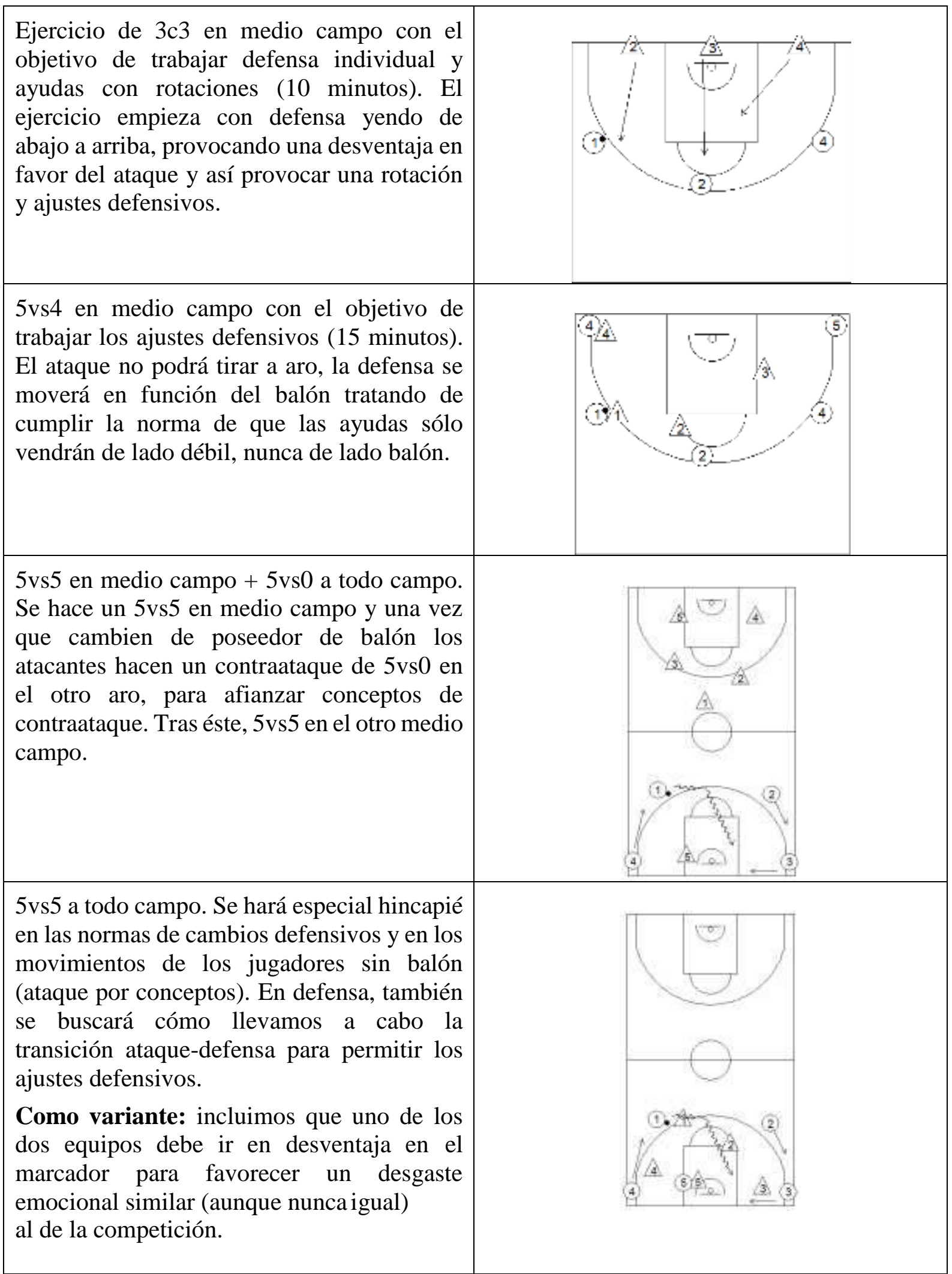




\section{OBJETIVOS/CONTENIDOS PRINCIPALES DE LA SESIÓN DEL MIERCOLES}

Adquisición de los mecanismos individuales de juego

Inicio de la recuperación activa

Elevada tensión pero ejercicios de muy corta duración

Entrenamiento con poca imprevisibilidad, pocos jugadores y espacios reducidos

Poco desgaste emocional (poco trabajo estratégico o de táctica colectiva)

En este entrenamiento, se buscará la mejora de los mecanismos individuales de los jugadores correspondientes a los principios que hemos tratado de potenciar durante la semana de entrenamientos.

\begin{tabular}{|c|c|}
\hline CALENTAMIENTO (15 MINUTOS) & REPRESENTACIÓN GRÁFICA \\
\hline $\begin{array}{l}\text { Ejercicio de } 2 \mathrm{c} 2 \text { para trabajar el balance } \\
\text { defensivo y activación del organismo. Para } \\
\text { ello, metemos una variante al ejercicio de } 2 \mathrm{c} 2 \\
\text { con balance defensivo. En esta ocasión los } \\
\text { defensores pasarán a atacar y los atacantes en } \\
\text { lugar de hacer el balance defensivo tendrán } \\
\text { que dar la mano a jugador de banda que serán } \\
\text { los que bajarán a realizarlo ( } 10 \text { minutos) }\end{array}$ & 4 \\
\hline $\begin{array}{l}\text { Ejercicio de finalizaciones previa salida } \\
\text { explosiva desde parado en el triple, } \\
\text { lanzándose el balón ( } 5 \text { minutos). Máximo } 2 \\
\text { botes dentro de la zona de tres puntos. Se } \\
\text { trabajarán finalizaciones con uno y dos } \\
\text { apoyos y salidas directa, cruzadas y en } \\
\text { reversos. }\end{array}$ & \\
\hline PARTE PRINCIPAL (50 MINUTOS) & REPRESENTACIÓN GRÁFICA \\
\hline $\begin{array}{l}\text { Ejercicio de } 3 \text { defensas consecutivas ( } 2 \\
\text { exteriores y una en poste medio) para trabajar } \\
\text { en función de los diferentes ajustes } \\
\text { defensivos que se hagan con cambios, tras } \\
\text { balance defensivo, etc. ( } 8 \text { minutos) }\end{array}$ & \\
\hline
\end{tabular}




\section{- Cíncia Digital}

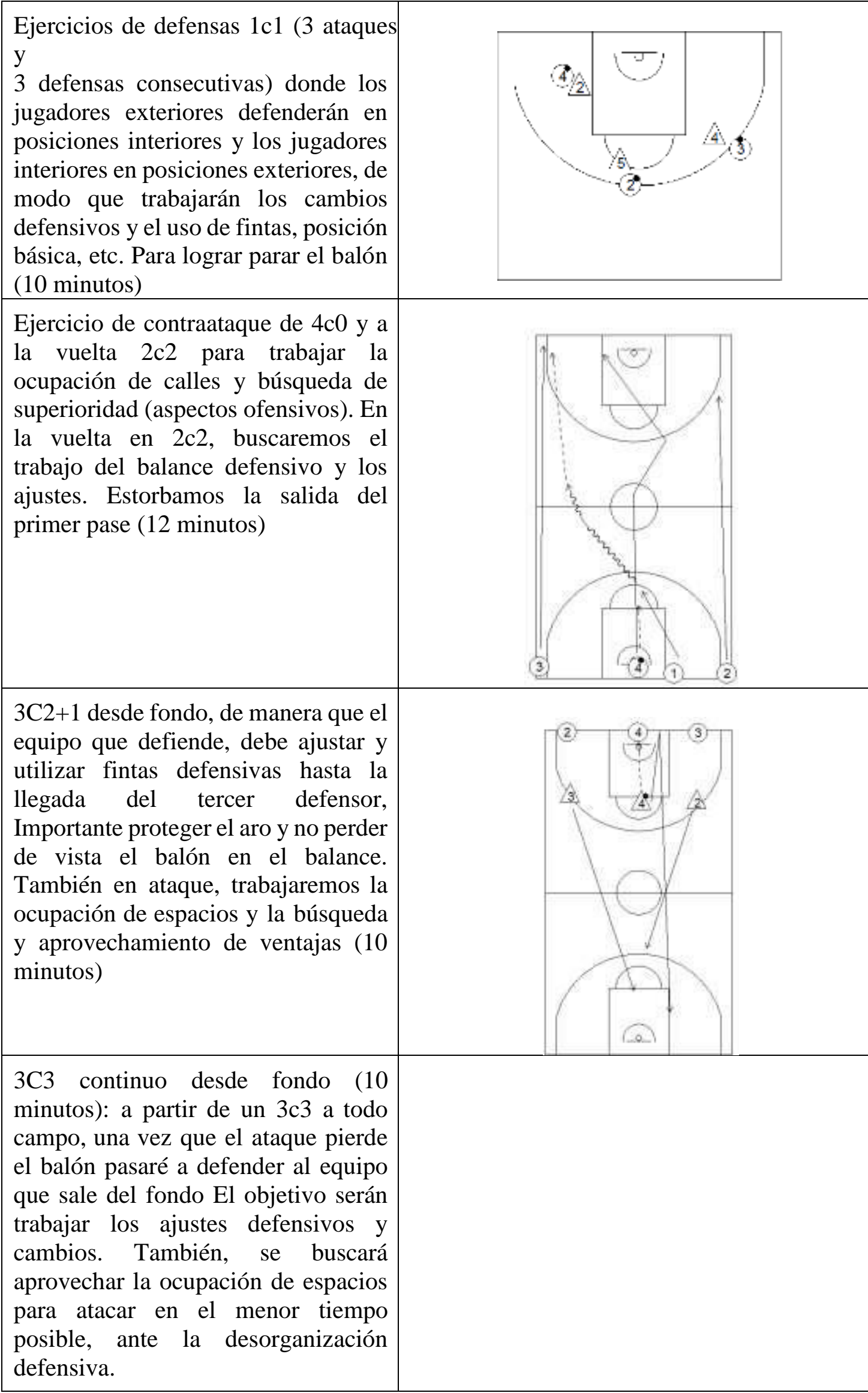




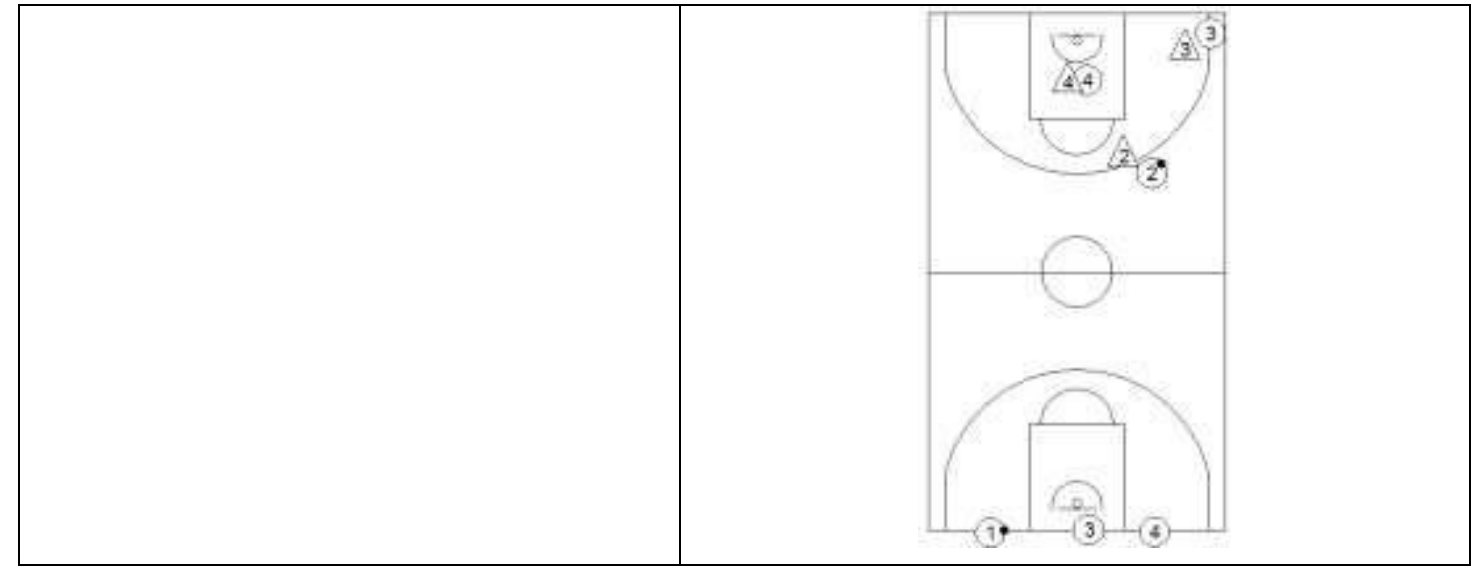

\section{OBJETIVOS/CONTENIDOS PRINCIPALES DE LA SESIÓN DEL JUEVES}

Recuperación activa para la competición

Trabajo de aspectos táctico-estratégicos fundamentales (macroprincipios)

Conocer características en vídeo del equipo rival

Recuerdo de sistemas de fondo y banda en situaciones de 5vs0 o $5 \mathrm{vs5}$ con defensa pasiva

Este entrenamiento es el previo a la competición, por lo tanto, el desgaste debe ser bajo y debe primar el trabajo del componente estratégico y de recuperación.

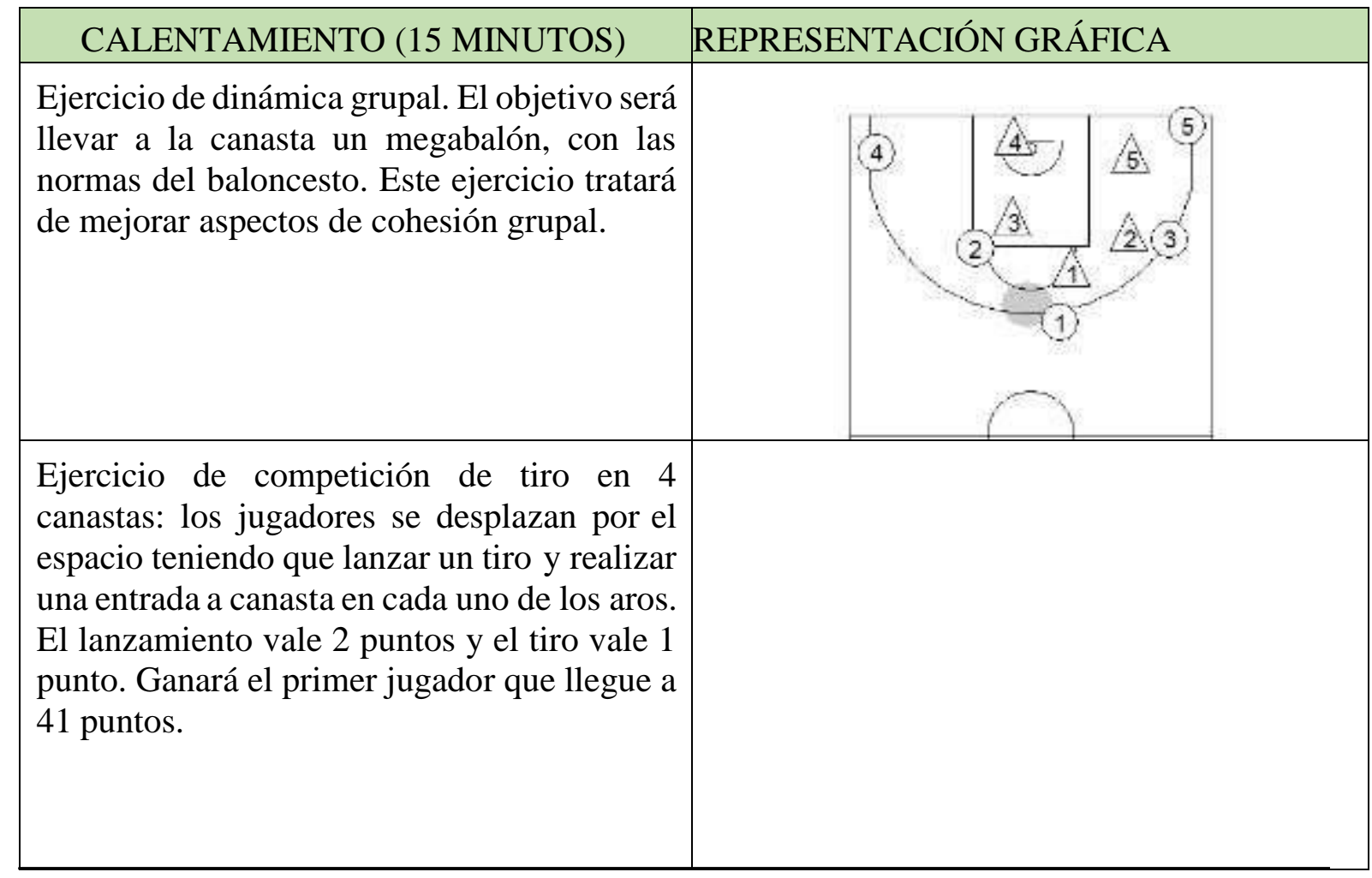




\begin{tabular}{|l|l|l|}
\hline &
\end{tabular}

\begin{tabular}{|l|l|l}
\hline $\begin{array}{l}\text { podemos cambiar o modificar de } \\
\text { nuestro jugar (10 minutos) }\end{array}$ & \\
\hline
\end{tabular}

Ejercicio de $5 \mathrm{vs} 0$ para trabajo de movimientos de ataque en media pista. Se puede incluir defensa y trabajar a partir de la defensa que hace el rival (10 minutos) 


\section{Ciencia

Inclusión de algún sistema de banda o fondo o recuerdo de los anteriores. Podemos dar nuevas pautas en función de cómo hemos visto que defienden las diferentes situaciones nuestros rivales ( 8 minutos) 


\section{CONCLUSIONES:}

- Lo más deficiente desde el punto de vista metodológico se centra en la atención del nivel de responsabilidad y compromiso de los jugadores para atacar, defender y realizar transiciones tanto de ataque como de defensa en superioridad numérica, en ocasiones no se tiene en cuenta estos sucesos para tener un dominio global del juego .

- La propuesta de morfociclo ofrece objetivos que guían a las deportistas en dirección de producir juego global en cuanto a organización defensiva, organización ofensiva, transición ataque /defensa y transición defensa /ataque, además de prepararlos para que puedan hacer emerger su creatividad a la hora de solucionar las incertidumbres presentes en el juego.

- Los resultados con la aplicación de la propuesta contribuyo a:

- Un aumento en la capacidad de utilizar lo aprendido en el entrenamiento y en el juego.

- Un aumento en la fuerza, la resistencia y rapidez de los deportistas producto del arrastre dejado por la aplicación de la periodización táctica.

\section{Referencias Bibliográficas}

Arjol, J.L (1997).Análisis sobre tareas integrales en el entrenamiento del futbol de alta competición training futbol, 11, 17,27.

Loaiza P.G.(2017).Periodización táctica. Planificación de una temporada en baloncesto de alto nivel (2017).

Morcillo, J.A, Cano, O, Martínez D.(2006).El valor de lo invisible .Fundamentación y propuesta de organización y entrenamiento específico del futbol.EFDeportes.com, revista digital, Nro. 92.http//www.efedeportes.com/efd92,htm.

Morente, J.S, Frauda V.L. (2012). Aplicación de la teoría de los sistemas dinámicos al entrenamiento deportivo: Futbol.EFDeportes.com, revista digital, Nro 165, febrero 2012, http.//efedeportes.com.

Oliveira, B, Almeiro, N, Resende, N .y Barreto.(2006).Mourinho, Porque tantas Victorias? Lisboa. Ed. Gradiva. 
Peñalver, O (2009).Emociones colectivas. Barcelona. Ed Alienta.

Seirul-lo, F. (2003).Sistemas dinámicos y rendimiento deportivo en deportes de equipos. Ist. Meeting of complex and sport INEFC. Barcelona.

Tamarit, X (2007) ¿Que es la periodización táctica? Vivenciar el juego. Pontevedra: Ed. Gradiva.

Torrents Martin, C(2005).La teoría de los sistemas dinámicos y el entrenamiento deportivo. Tesis doctoral INEFC-Barcelona. Lleida. 


\section{PARA CITAR EL ARTÍCULO INDEXADO.}

Alzola Tamayo, A., Ortiz Fernández, D., Vaca, M. E., \& Alzola Tamayo, S. (2019). Determinación estructural de la periodización táctica en el baloncesto femenino de la Espoch sede Morona Santiago Ecuador. Ciencia Digital, 3(3.1), 390-408. https://doi.org/10.33262/cienciadigital.v3i3.1.710

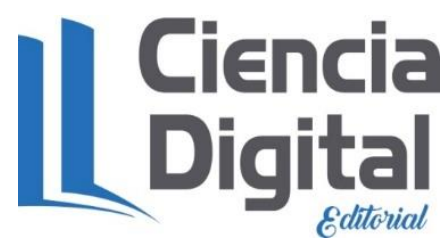

El artículo que se publica es de exclusiva responsabilidad de los autores y no necesariamente reflejan el pensamiento de la Revista Explorador Digital.

El articulo queda en propiedad de la revista y, por tanto, su publicación parcial y/o total en otro medio tiene que ser autorizado por el director o editor de la Revista Explorador Digital. 